\title{
Resumen
}

El presente artículo presenta la proponer la mejora de la eficiencia en la gestión de las actividades operacionales de una empresa de servicio, permitiendo disminuir los costos asociados a los procesos, en pro de mantener la planta de recursos humanos, identificando el nivel de compromiso que tienen los trabajadores con la empresa y su disposición a nuevos cambios organizacionales, examinando y describiendo los procesos operacionales, identificando aspectos críticos en los procesos operacionales y generando propuestas de mejoramiento y monitoreo de los procesos. En definitiva, a través de los resultados, se determina que la empresa que acoge las propuestas de mejoramiento estará preparada para enfrentar alguna eventualidad, ahora si la empresa decide no prepararse con anterioridad, es decir, prefiere ser reactivo antes que proactivo decidirá en el momento justo tomar medidas respecto a la situación que la aqueja.

Palabras Claves: Eficiencia, Gestión, actividades operacionales, cambios organizacionales, procesos operacionales, monitoreo.

\section{MEJORAMIENTO EN LA GESTIÓN DE ACTIVIDADES OPERACIONALES Y LA MOTIVACIÓN DE LOS RR.HH DE LAS EMPRESAS.}

\section{IMPROVEMENT IN THE MANAGEMENT OF OPERATIONAL AC- TIVITIES AND MOTIVATION OF HUMAN RESOURCES IN EN- TERPRISES}

\author{
Connie Álamos Núñez ${ }^{1}$
}

\begin{abstract}
This article proposes that an improvement in management efficiency of the operational activities of a utility allows to diminish the costs associated to processes in favour of maintaining its human resources and identifying the level of compromise that workers have with their enterprise and their willingness to adapt to new organizational changes. To achieve this it is necessary to examine and describe the operational processes identifying critical aspects in them and making improvement proposals and continuous monitoring of the processes. Definitely, the results have determined that an enterprise that embraces the improvement proposals will be better prepared to face any difficulty, but if the enterprise decides not to be prepared in advance, that is to say, prefers to be reactive rather than proactive will have to take action on a difficult situation when that happens.
\end{abstract}

Key Words: Efficiency, Management, operational activities, organizational changes, operational processes, monitoring.

'Alumna Tesista de la Carrera de Auditoría de la Universidad de Valparaíso, teniendo como profesor guía al Prof. Iván Cubillos G. 


\section{INTRODUCCIÓN.}

Se vive en un mundo globalizado por lo que, lo que ocurre en un país tiene consecuencias en los otros, con una mayor 0 menor intensidad. Existiendo esta relación directa entre países, las entidades deben reflexionar y analizar vivencias pasadas para establecer de qué manera debe enfrentar una nueva dificultad, que puede ser económica, política, social o natural. Cuando cualesquiera de estos factores actúa perjudicando al sistema económico de la región donde se originó la problemática, las organizaciones locales deben estar en alerta y preparadas para enfrentar de manera exitosa la crisis que se aproxima, según expresa EFE (2011).

Cuando la recesión es inminente, el mundo empresarial comienza a elaborar estrategias para enfrentar la crisis y en los últimos años los "Despidos Masivos" están siendo la alternativa más utilizada para reducir costos, ya que las remuneraciones son uno de los costos más elevados que tienen las empresas, así menciona Chiavenato (2002); y reducir éstos es la manera más sencilla de lograr un resultado favorable. Lo que las organizaciones no ven es que esta es una práctica con la que se obtienen beneficios al corto plazo, lo que conllevará a un mayor costo en el futuro, pues cuando la economía mejore, se deberán realizar nuevamente contrataciones para elevar el nivel de actividad y así generar mayores ingresos, según Gómez, Balkin y Cardy (2001).

Por lo tanto, el presente artículo presente el análisis de los procesos operacionales de una organización, a través del estudio de un diagrama de flujo, en donde se identificarán instancias por mejorar. 


\section{DESARROLLO DEL TRABAJO.}

Un aspecto importante en las empresas, es la motivación que entregan los niveles superiores a sus trabajadores, es por esto que debemos definir en qué se basa.

\section{MOTIVACIÓN.}

La motivación según Hitt, Black, Porter, (2006) son los impulsos que guían a un individuo a realizar ciertas acciones en el día a día.

Según este mismo autor, se puede clasificar la motivación en tres puntos, reconocidos como:

Características Individuales: Estas dependen de cada persona y se basan en sus ideales y necesidades. Ellas los motivaran a realizar las actividades que se propongan; a su vez, pueden influir en el desempeño y perfeccionamiento profesional que tenga las personas.

\section{Características del puesto de trabajo:}

Se relaciona con el reconocimiento que recibe una persona por el trabajo realizado. Esto provocará una motivación para seguir avanzando y mejorando, a medida que va ejerciendo sus labores al interior de la empresa.

\section{Características de la situación lab-} oral: Esta categoría se caracteriza por estar destinada a los miembros de la organización, reconociéndose ciertas acciones como el pago de remuneraciones, la entrega de capacitaciones e incentivos, por alcanzar cierto nivel de actividad.

\section{CAMBIOS ORGANIZACIONALES.}

Según Manuel Mariña (2007): las or- ganizaciones que logran cambiar son flexibles lo que les genera una ventaja competitiva frente a las que no lo son. Por otro lado, los cambios que son planeados por las organizaciones tienen la intención de mejorar equipos y áreas de trabajo al interior de la empresa logrando optimizar significativamente. Es importante que cuando se decida realizar un cambio, los miembros de la empresa conozcan la necesidad y razones de esta medida para que exista una disposición por parte de ellos en cambiar su actuar cotidiano, permitiendo lograr el cambio sin mayores problemas, ya que si no existe la intención, será muy difícil realizar la implementación.

Este mismo autor identifica algunos aspectos que son reconocibles cuando un trabajador se resiste al cambio propuesto por la empresa, ellos son:

Percepciones: Se vincula con como creen que les afectara el cambio en sus vidas y de solo imaginar el posible impacto que tendría unirse a estas nuevas medidas, se resistirían a él.

Personalidad: Algunos personas por su personalidad pueden influir a trabajadores vulnerables y con poca decisión, por eso es importante identificar quienes son esas personas rígidas en sus pensamientos y quienes son aquellas susceptibles de ser influenciados. De este modo, la empresa sabrá con quienes debe realizar un trabajo previo mayor para que se unan al cambio.

Amenazas al poder y la influencia: Para algunos trabajadores, tener influencia sobre sus pares es agradable, lo que puede ser un punto a considerar al momento de identificar quienes se resistirían al cambio.

Temor a lo desconocido: Cuando las personas se enfrentan a algo desco- 
nocido, provoca duda e inseguridad. Provocando un rechazo a esta nueva medida.

\section{COMUNICACIÓN.}

Según Livacic, C, (2009) la comunicación es un medio que utilizan las organizaciones para simplificar las tomas de decisiones, obtener respuestas y entregar información a la comunidad, acerca de, las metas de la organización 0 cambios por realizar.

De acuerdo Livacic (2009) la comunicación es requerida para los siguientes casos :

- Establecer y difundir las metas de una empresa.

- Desarrollar planes para su logro.

- Organizar los recursos humanos y de otros tipos en la forma más efectiva y eficiente.

- Seleccionar, desarrollar y evaluar a los miembros de la organización.

- Dirigir, orientar, motivar y crear un clima en el cual las personas quieran contribuir. - Controlar el desempeño

\section{OPERACIONES.}

Las operaciones son parte de un sistema de transformación. Cuando se habla de un sistema, se relacionan las entradas, los procesos y las salidas. Estas fases se ven afectadas por aspectos internos y externos, los que influirán al momento de determinar cuál es el proceso apropiado para cada actividad desarrollada por la empresa, esta expresión se ve demostrada en la figura $\mathrm{N}^{\circ}$ 1: La visión integrada de un sistema de operaciones. El ambiente interno está dado por aquellas áreas que conforman a una organización y el externo por factores que interactúan con la organización. Los constantes cambios a los que están expuestas las empresas, consecuencia de la globalización, afectan los procesos, esto quiere decir, que ellos deben ser flexibles y adaptables a las necesidades que se generen en un momento determinado.

\section{Figura $n^{0}$ 1: La visión integrada de un} sistema de operaciones

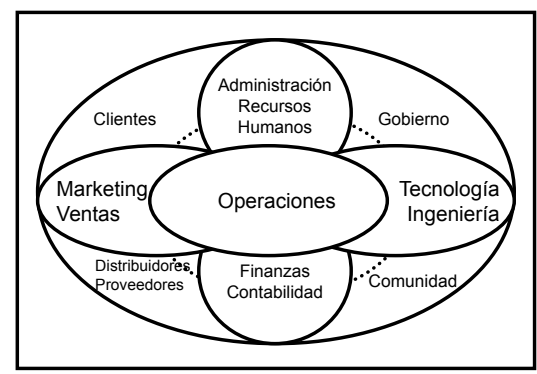

Fuente: J. Heim y W. Compton (1992) referenciado en Zúñiga (2005)

\section{TIPOS DE PROCESOS.}

Los procesos son distintos en cada empresa, se adaptan a cada requerimiento y necesidad que se genere. A su vez, existen tipos de procesos, que son la estructura básica de aquel proceso que la entidad seleccione para desarrollar su actividad generadora de productos o servicios, este siempre debe ser un apoyo para la estrategia de flujos. A modo de referencia, cuando se crean productos personalizados, el proceso que más le favorece es el proceso en una línea o por lote. En cambio, cuando se produce en grandes cantidades y estándares, se asocia a un proceso de línea de ensambles.

\section{Procesos de producción}

Según Schroerder (2005) para las empresas productoras existen dos cla- 
sificaciones de los procesos uno que se denomina por flujo de productos y otro que se denomina por pedidos de clientes. Ambos poseen su propia clasificación. El primero se sub clasifica: en línea, por lote, por proyecto. El segundo se sub clasifica por pedidos, por inventarios.

\section{Flujos de productos}

Las empresas productoras se relacionan con los flujos de productos. Las empresas que se dedican a la prestación de servicios no se asocian a este tipo de flujo, pero sí, al que se denomina flujo de clientes 0 de información. Para la primera clasificación se tiene:

- Flujo en línea: las operaciones son contínuas, existen etapas en las que el producto debe avanzar de manera secuencial, desde la primera etapa a la última, sin excepción. Si el producto no cumple con todos los niveles, el resultado obtenido será defectuoso e inutilizable. Las operaciones que se realizan en cada fase son siempre las mismas, no sufren cambios. En este proceso los productos se crean en grandes volúmenes y estandarizados, lo que permite decir que se trata de una clasificación poco flexible. Ejemplo de esta clasificación son: automóviles, computadoras, restaurantes de comida rápida y cafeterías.

Figura $n^{0}$ 2: Flujo en línea

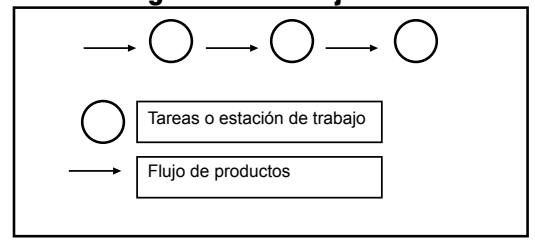

Fuente: Schroerder (2005)
Flujo por lote: las operaciones, en este tipo de flujo, son similares y dependiendo del proceso en el que ingresan los insumos, es el producto que se obtendrá. Esto quiere decir que existirá una diversidad de productos al final de cada centro de trabajo. Estos pueden ser mejorados para que se realice un nuevo tipo de producto, siempre y cuando la empresa cuente con una capacidad flexible. Los volúmenes de fabricación que se tiene en este tipo de flujos son medios. Algunas actividades que se relacionan con este tipo de flujo son: programación de un viaje en avión, procesamiento de préstamos hipotecarios y otros.

Figura $n^{0} 3$ : Flujo por lote

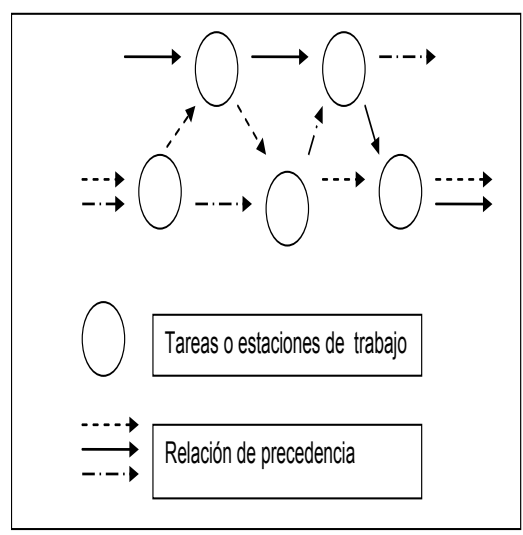

Fuente: Schroerder (2005)

- Flujo por proyecto: en este caso, las tareas o productos confeccionados son únicos, es decir, no hay otro igual, por lo que las empresas deben contar con trabajadores especialistas en la tarea a desarrollar, mientras que las instalaciones pueden ser de uso general. Es un trabajo realizado en base a los re- 
querimientos del cliente $y$ en volúmenes reducidos, a modo de ejemplo, están la planificación de un evento importante, la construcción de un hospital y producción de aeronaves, entre otros.

Figura $\mathrm{n}^{0} 4$ : Flujo de proyectos

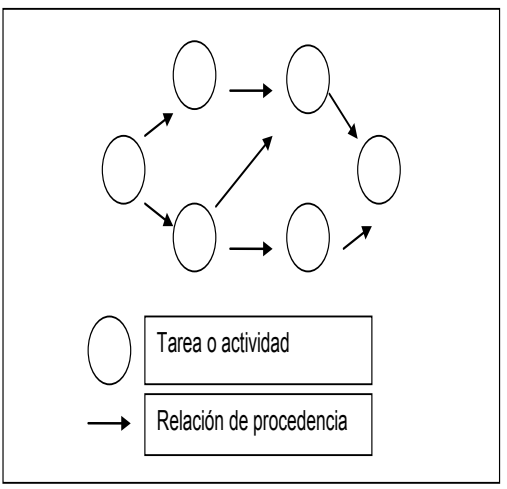

Fuente: Schroerder (2005)

\section{Procesos de servicios.}

Schroeder (2005) habla de servicios cuando se está en presencia de una actividad que se realiza y consume al instante. Esto quiere decir, que el cliente debe estar presente en el momento que se ejecuta el trabajo encomendado, lo que provocará que el proceso se vuelva inestable por las posibles críticas que puede realizar en su desarrollo. Esta sencilla definición es considerada la más apropiada y representativa, ya que, adicional a ésta, se dice que un servicio es un producto intangible, lo que quiere decir, que no se puede definir ni medir fácilmente.

A partir de Chase, Jacobs y Aquilano (2005) se rescata que la organización que se dedique a la prestación de servicios debe considerar cuatro elementos fundamentales que son: quién será el cliente al que desean llegar, cómo se diferenciará de los otros servicios similares, cuál será la estrategia del servicio y, por último, qué instalaciones, personal y otros se requerirá para efectuar el servicio.

Al ocurrir alguna falla en el servicio, se debe recurrir a una recuperación inmediata u oportuna, ya que si no es solucionada a tiempo, se producirá la insatisfacción por parte del cliente, quién no volverá a solicitar el trabajo. En cambio, si la anomalía tiene solución, éste aceptará lo ocurrido y podrá quedar conforme con la solución.

\section{Contacto con el cliente.}

El contacto con el cliente según Chase, Jacobs y Aquilano (2005), se produce cuando el cliente participa presencialmente en la prestación del servicio. En la creación de un servicio, puede ocurrir que exista un mayor o menor contacto con el cliente, lo que provocará ciertas diferencias que son importantes de considerar al momento de diseñar el proceso. Estas características serán clasificadas dependiendo del grado de contacto, en primera y segunda clasificación.

La primera clasificación se da cuando hay poco contacto entre la empresa que presta el servicio y el cliente a quien se le concede el servicio. Las características de esta relación son:

- No se requiere una
interacción cara a cara
- Necesitan de em-
pleados con habilidades técni-
cas, rutinas eficaces y estan-
darización, tanto del producto
como del proceso.
- Las operaciones pu-
eden funcionar hasta niveles
promedios de demanda.
- El cliente solo se in-
teresa en las fechas de termi-
nación.
interacción cara a cara pleados con habilidades técnicas, rutinas eficaces y estandarización, tanto del producto como del proceso. eden funcionar hasta niveles promedios de demanda. teresa en las fechas de terminación. 
- La producción, que puede determinarse, permite sistemas de salarios basados en la misma.

(Chase, Jacobs y Aquilano, 2005)

Por otra parte, la segunda clasificación, cuando hay mucho contacto con el cliente, basándose en los autores ya mencionados las características son las siguientes:

- $\quad$ La demanda del cliente es cambiante 0 incierta.

- Los empleados deben ser flexibles, agradables y tener disposición para trabajar con el cliente.

- Entregar una respuesta inmediata cuando la demanda se presenta en situaciones límites.

- $\quad$ Requieren precios más altos y mayor fabricación a la medida.

- El cliente está en el programa de producción y debe adaptarse.

- $\quad$ La producción variable requiere sistemas de salarios basados en el tiempo. lano, 2005)

(Chase, Jacobs y Aqui-

\section{Diseño del servicio}

Según Schroeder (2005) se pueden destacar cuatro tipos de servicios que se reconocen como: fábrica de servicios, taller de servicios, servicios masivos y servicios profesionales.

\section{Fábrica de servicios}

Este tipo de servicio se caracteriza por entregar un servicio basado en un modelo que es poco adaptable a cada cliente, por esta razón es que se requiere de menos trabajadores brindando un servicio poco diferenciador. Las empresas que se identifican en esta categoría son hoteles, líneas de aviación y otros. (Schroeder, 2005)

\section{Taller de Servicios}

En este ítem, la participación del cliente es mayor, por eso se dice que el producto del servicio estará más adaptado e integrado con los requerimientos del cliente. En este punto, la mano de obra está presente en un menor grado. Ejemplos de esta categoría son hospitales, servicios de reparación y otros. (Schroeder, 2005)

\section{Servicios masivos}

En este tercer ordenamiento, la personalización del trabajo es baja, a diferencia de la intensidad de la mano de obra que es elevada, lo que provoca una baja en la automatización $y$, por ende, una baja en la eficiencia de la conversión. Algunos ejemplos que representan la clasificación son: escuelas y ventas al mayor, entre otras instituciones. (Schroeder, 2005)

\section{Servicios profesionales}

En este caso, la automatización, y estandarización del servicio es mínima, lo que provocará una escasa eficiencia, ya que se requiere de una interacción significativa con el cliente. Adicional a esto, es necesario tener un elevado grado de intensidad de la mano de obra. En esta categoría, se caracterizan los profesionales como médicos y contadores, entre otros. (Schroeder, 2005)

Según ha establecido Chase, Jacobs y Aquilano (2005), el diseño de los servicios, se representa por tres categorías: el método de línea de producción, el método del auto servicio y, por último, el método de la atención personal.

\section{MEJORAMIENTO DE PROCESOS.}

Cuando una empresa decide realizar 
mejoramientos en sus procesos, Io que está buscando es mejorar "la calidad, el tiempo de procesamiento, los costos, los errores, la seguridad o la puntualidad en la entrega" (Krajewski y Ritzman, 2000:110), lo que se logrará, realizando un análisis de las actividades que desarrollan los trabajadores. Éstas deben ser comprendidas y cuestionadas en su amplitud y detalle. Para obtener un resultado de calidad se debe observar continuamente las áreas que son susceptibles de tener desviaciones $y$, por ende, que no cumplan con los objetivos que se han establecido para dicha tarea.

Cuando el analista tiene todas las áreas del proceso estudiadas, puede tomar decisiones para mejorarlos con soluciones diversas. Todo depende de los requerimientos y estrategias que la empresa establezca para entregar sus productos o servicios, de la manera más apropiada y oportuna. Algunas de estas soluciones pueden ser: simplificación de las tareas, disminución en el requerimiento de materiales, cambio de proveedores o simplemente suprimir alguna actividad que no entregue valor al resultado de una serie de procesos y labores realizadas por el personal de la institución.

Según Krajewski y Ritzman (2000) las características que tiene un proceso que requiere de mejoramientos son:

- $\quad$ El proceso es lento en responder a los deseos del cliente.

- $\quad$ El proceso origina demasiados problemas 0 errores en relación con la calidad.

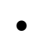

El proceso es

costoso.

- $\quad$ El proceso es un cuello de botella y el trabajo se acumula en espera de llegar al final.

El proceso

conlleva trabajo desagradable, contaminación 0 poco valor agregado".

(Krajewski y Ritzman, 2000, 110)

Existen varias técnicas para analizar los procesos, pero en esta oportunidad se describirá el diagrama de flujo, que es considerado como una técnica básica para realizar esta actividad. El analista debe cuestionarse una serie de características que son propias del proceso como los que menciona el autor ya señalado "¿Qué se está haciendo?, ¿Cuándo se hace?, ¿Quién lo está haciendo?, ¿Dónde se está haciendo?, ¿cuánto tiempo se requiere? Y ¿Cómo se está haciendo?" (Krajewski y Ritzman, 2000: 110) Con posterioridad a este cuestionamiento, se debe detallar los elementos de cada proceso y se obtendrá una amplia visión de lo que se está realizado para desarrollar el producto 0 servicio. Finalmente, se debe determinar los cambios que ocurrirán en dichos procesos, producto del análisis crítico de ellos.

\section{Diagrama de flujo}

Este es un esquema que permite identificar todas las etapas de un proceso. Es creado por el mismo analista, pues no tiene una estructura determinada, solo depende de cada proceso presente en la empresa. Se puede diseñar en base a dos criterios: el de diagrama simple, que contiene recuadros, flechas y líneas, indicando cada fase que se reconoce; y otro, más complejo, que requiere mayor elaboración. Se agrega un dibujo real de la planta de trabajo, incorporando todos los movimientos que realizan los encargados de ese proceso. Junto a cada recuadro es apropiado señalar 
algunas mediciones, que pueden entregar información relevante como: tiempo, calidad, errores, capacidad 0 costos. Datos que son mencionados en Krajewski y Ritzman (2000).

Dentro de este diagrama se encuentran las actividades que son visibles para los clientes y aquellas que solo ocurren para ojos de la empresa. Esto quiere decir que se evidencia, desde el primer contacto con el cliente, hasta cuando el cliente hace uso o retiro del producto. 
Figura $\mathbf{N}^{\circ}$ 5: Diagrama de Flujo

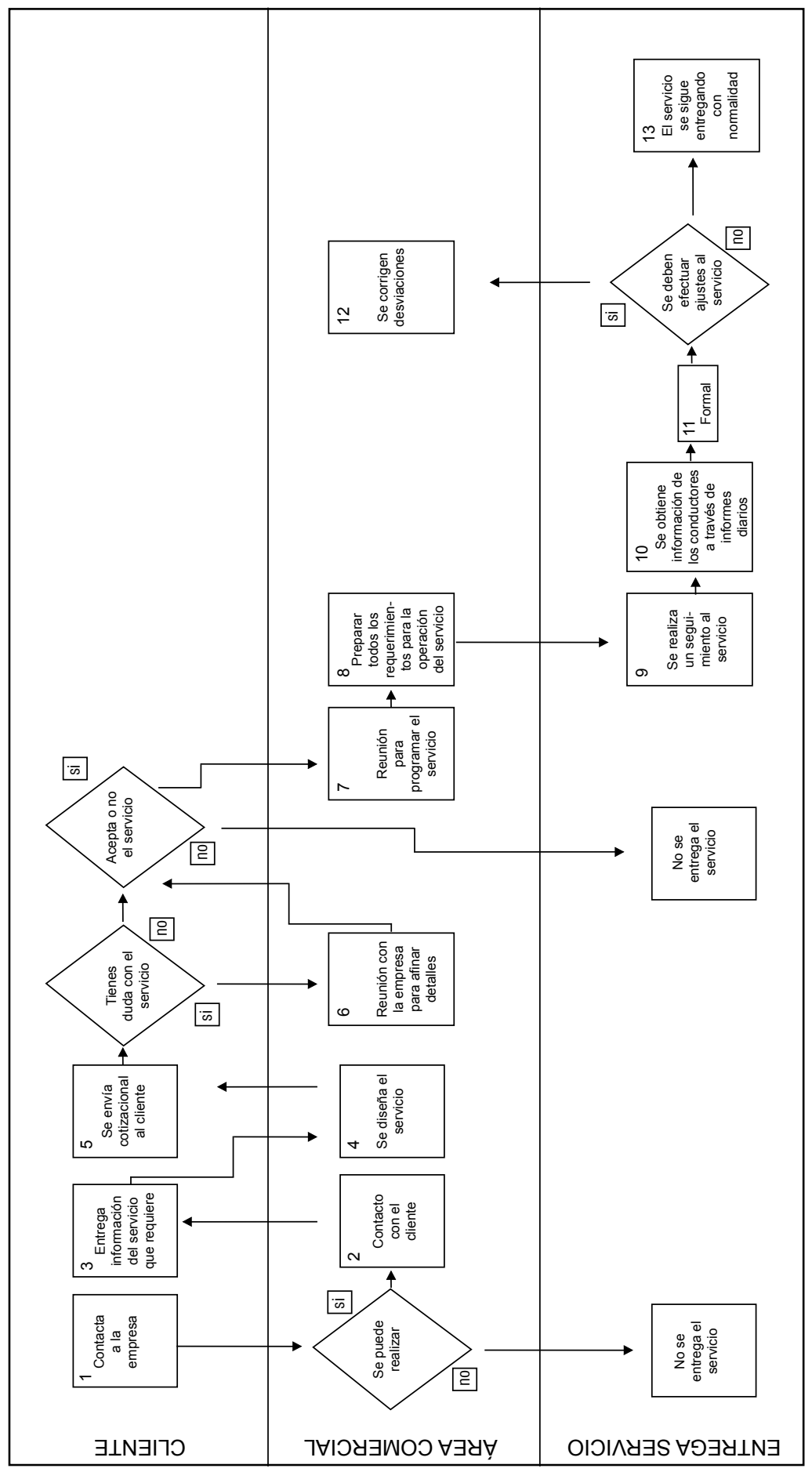

Fuente: Krajewski y Ritzman (2000) 


\section{METODOLOGÍA.}

La metodología utilizada en la presente investigación, corresponde a un análisis cualitativo.

Los criterios que se emplearán para ordenar la información recopilada, son:

Motivación, cam-
bios organizacionales y comu-
nicación.
- $\quad$ Estructura de costos, que nacen producto de la actividad principal u otros que nacen por ser actividades indirectas a la principal.

- Las operaciones mismas dentro de una organización, como lo es el tipo de proceso y sus cualidades.

- $\quad$ Criterios que se utilizarán para apoyar el mejoramiento de los procesos operacionales.

El Instrumento, que permite la identificación del nivel de compromiso que tienen los trabajadores con la empresa, es el cuestionario.

La investigación se llevará a cabo por sobre la base de lo expuesto en: Revista Venezolana de Gerencia v.13 n.41 Maracaibo mar. 2008 titulada "La responsabilidad social en la industria farmacéutica del Estado de Zulia", con algunas modificaciones $\mathrm{e}$ incorporaciones de temáticas nuevas.

El cuestionario se aplicará a
los trabajadores de la empresa,
considerando una muestra representativa de ellos. La muestra será determinada de acuerdo al programa Stats, considerando un $5 \%$ de error, un $95 \%$ de confianza, y un $99 \%$ de variabilidad.

Al evaluar la confiabilidad del instrumento el programa determina que sí es confiable, ya que en el ítem Cronbach's Alpha se obtuvo 0,796 y lo mínimo que se debe tener, para que sea confiable, es 0,5 .

\section{RESULTADOS.}

La comunicación que se genera en la empresa entre quienes la dirigen y sus conductores es adecuada y diaria, ya que tienen diversas instancias en donde se da a entender todo el funcionamiento de la empresa, los requerimientos del servicio y, con posterioridad a la entrega del servicio.

Habitualmente se consideran las opiniones de los conductores, ya que existe una vía de comunicación formal e informal, donde ellos entregan sus opiniones 0 sugerencias, respecto al servicio. Luego, el encargado de transporte las analiza y deriva al área que se encarga de la situación que está ocurriendo para darle solución a ellas.

El conductor cuenta con todas las herramientas necesarias para que el cliente quede satisfecho con el servicio y no se generen problemáticas en su desarrollo. Estas pueden ser: ubicaciones, mapas, aunque generalmente se designan a conductores que conozcan la zona para disminuir la probabilidad de que se genere algún inconveniente. En el caso que el conductor tenga dudas, porque no se le entregó toda la información, se entregan referencias y existe un contacto telefónico, en caso de necesitar ayuda, mientras se entrega el servicio.

Para una empresa es importante que sus trabajadores conozcan y entiendan los objetivos que se espera alcanzar, ya que todos los miembros deben aportar algo de si. Para los conductores, conocer sus beneficios es importante, 
ya que de esta manera podrán gozar de ellos sin ningún problema. La empresa se preocupa de entregar esta información, desde el primer día que se incorporan los conductores a formar parte del equipo de trabajo.

Brindar estabilidad laboral a los conductores es esencial, ya que ellos son quienes representan a la empresa cuando el servicio se está entregando, si ellos se sienten estables en el trabajo su desempeño será el esperado. En cambio, si se trabaja en un ambiente de incertidumbre existe la posibilidad que el conductor no desarrolle sus actividades al 100\%, como debe ser. Por esta razón es que la empresa se preocupa de que el conductor esté cómodo y contento con su trabajo.

Existe una preocupación por parte de la empresa para que sus conductores estén preparados al momento de prestar el servicio, de esta manera contarán con los conocimientos y habilidades que les permitirán desarrollar sus labores de manera óptima. Siempre manteniendo una relación con los estándares definidos en las políticas de calidad.

Sí se genera un ambiente de confianza entre el jefe y los conductores, pues las instancias están a disposición de quienes quieran y requieran utilizarlas, siempre y cuando se siga con el conducto regular de la empresa. Esto quiere decir que el conductor que necesite hablar con su jefe u otro es porque en primera instancia el supervisor no puedo dar respuesta a la pregunta que se tenía.

Cada vez que se generan cambios la empresa los informa a sus conductores y trabajadores en general, a través de charlas, capacitaciones, si son temas que afecten directamente a los conductores y otras instancias de diálogo en donde se les explica por qué ha ocurrido esta situación y cómo se enfrentará.

En definitiva, la empresa considera que la comunicación es fundamental para cumplir con las metas y servicios que deben alcanzar y entregar. Por esta razón, es que se fortalece diariamente esta instancia, ya que es importante dar a conocer sus necesidades y escuchar las opiniones que tienen los conductores, logrando responder, de manera dinámica y oportuna, a todos los requerimientos que se presenten a lo largo de la actividad.

La motivación de los conductores debe ser buena, realizar sus tareas no deberia ser bajo un escenario de amargura y desmotivación. Al contrario, la empresa entrega seguridad, estabilidad y capacitaciones, con el fin que sus conductores entreguen un buen servicio y sientan ser parte del grupo de trabajo. Los reconocimientos son importantes, ya que les entrega nuevas responsabilidades como nuevas maquinarias o servicios más importantes. La confianza se presenta diariamente entre la empresa y los conductores, lo que ayuda en la comunicación y motivacion de los mismos.

Desde que los conductores se incorporan a la empresa, se les está informando que pueden sufrir cambios en sus labores, a lo largo del tiempo, por lo que los conductores están preparados cuando ocurre una situación así. La empresa está comprometida con sus trabajadores, ya que han demostrado que se preocupan de ellos. En situaciones extremas, no los dejan excluidos ni menos sin trabajo. Se les explica todo lo que ocurre y en qué situación se encuentran, para que ellos formen parte de cada período y así trabajen juntos para enfrentar las situaciones compli- 
cadas.

\section{DISCUSIÓN DE RESULTADOS.}

Con la información obtenida de los cuestionarios realizados a la entidad se lleva a cabo una comparación entre estos y los aspectos teóricos tratados, recopilados de diversas fuentes bibliográficas, lo que permitirá identificar el perfil de la empresa sobre la cual se realiza esta investigación. Del análisis comparativo se desprenden las siguientes situaciones:

Según Hitt, Black, Porter, (2006) la motivación se sustenta en tres características. En esta investigación se reconocieron sólo dos, las de puesto de trabajo y situación laboral.

Esta empresa motiva a sus conductores reconociéndolos verbalmente por el trabajo realizado y, además, mejoran los vehículos que utilizan diariamente. En este punto, se da una diferencia con la información entregada por los conductores, ya que ellos consideran que solo algunas veces se realiza un reconocimiento. Eso implica que puede generarse una desmotivación a nivel de los conductores.

Relacionado con la situación laboral, la empresa está constantemente capacitando a sus trabajadores y, por supuesto, pagando sus remuneraciones.

Según Manuel Mariña (2007) los cambios que realiza una organización, deben ser informados a la comunidad, para que ellos estén enterados y así se formen una expectativa de lo que ocurrirá entregando disposición a estas nuevas actividades.

En el caso de la empresa bajo estudio siempre informan a sus trabajadores cuando deciden realizar un cambio organizacional y sus conductores no tienen problemas en acogerlo, siempre que sea por una razón fundamentada. Pero bajo otras situaciones se deben considerar las características personales de cada uno, existen conductores que son rígidos en sus pensamientos, algunas veces influenciables y en caso de perder la influencia que tienen sobre otros algunas veces se resistirían. Por otro lado, la incertidumbre que se genera por enfrentar algo desconocido no es un impedimento para los conductores.

La comunicación al interior de esta empresa se relaciona fielmente con lo establecido por Livacic, C, (2009), que entregan los espacios para el diálogo y la información de situaciones 0 actividades que requieren que sus conductores conozcan.

Los conductores afirman que sí se difunden las metas y objetivos de la empresa, que se está valorando su trabajo, pero que hay deficiencias con las otras áreas que, algunas veces, son oportunas en responder. La empresa debe preocuparse de esta falencia.

Se logra identificar con la descripción de las actividades que se realizan en el proceso de servicio, que es el cliente quien entrega todos los lineamientos y requerimientos que estime apropiados para satisfacer sus necesidades. De esta manera, la empresa de transporte logrará diseñar el servicio, a base de los parámetros entregados por los posibles usuarios.

En esta empresa se registran tres tipos de costos significativos relacionados al transporte de pasajeros, son: Combustible, Remuneraciones e intereses por concepto de financiamiento. 
Estos costos se pueden clasificar según Jones, Werner, Terrel y Terrel (2001) en:

- Costos de materiales directos

- Costos de mano de obra

- Costos indirectos de servicios

Luego de identificar con qué tipo de proceso se relaciona la empresa e indagar respecto a las actividades que realizan para diseñar el servicio, se puede tomar la decisión de realizar mejoras, si es que la empresa considera que deben mejorar en calidad, tiempo o costos así lo menciona Krajewski y Ritzman (2000).

Según este mismo autor, cuando se realizan mejoramientos uno debe cuestionar las características que son propias del proceso. En el caso de esta indagación, los cuestionamientos principales fueron: ¿Qué se está haciendo?, ¿Cuándo se hace?, ¿Quién lo está haciendo y ¿Cómo se está haciendo?". Con estas interrogantes resueltas, se inicia un análisis tipo diagrama detallado con las actividades realizadas. Esto permitirá obtener una visión clara del trabajo realizado por la empresa, finalizando con el reconocimiento de las actividades que se podía intervenir, con una mejora.

Se consideraron tres tipos de operaciones relacionadas al plan de adquisiciones, combustible y gestión de costos. Se logró aportar, mejorando ciertas actividades como que se realicen cotizaciones y se controle el consumo de combustible. Si la empresa acoge estas actividades, contribuirá a un menor gasto en el consumo de los recursos. 


\section{CONCLUSIONES.}

Si la empresa acoge las propuestas de mejoramiento, les ayudará a estar preparados para enfrentar alguna eventualidad. Además, podrán decir que están preparados y no hay de qué preocuparse. Se debe considerar que las mejoras fueron identificadas a través de los resultados obtenidos por la aplicación de los instrumentos de análisis, por lo tanto, podrían existir otras alternativas.

El trabajo para diseñar e identificar las propuestas se basa, principalmente, en la confección de un diagrama de flujo (Figura $\left.N^{\circ} 5\right)$ en donde se reconoce cada una de las actividades que realiza la empresa, desde que el cliente la contacta hasta que el servicio es entregado. Luego de determinar cuáles son las actividades, se detalló cada una de ellas, explicando qué ocurre, quién la realiza, siguiendo una secuencia cronológica.

El compromiso que tienen los conductores con la empresa es positivo, esta aseveración se respalda con la buena comunicación y motivación que se genera al interior de la empresa. Queda pendiente reforzar ciertas instancias, como es el trabajo con otras áreas y el reconocimiento del trabajo realizado por ellos. Estas dos falencias no serán un impedimento en el momento que se decida realizar un cambio que afecte la labor de un conductor, por lo tanto, se puede asumir que en el caso de identificar nuevas mejoras, ellos los acogerían sin problema. Se reconoce que las mejoras propuestas no afectarán sustancialmente el trabajo realizado por los conductores, lo que permite asumir que no tendrán problemas en apoyarlas. Cuando se decide identificar el compromiso, por parte de ellos, con la empresa, es con el fin de saber cuál sería su actitud en caso que se decidan a realizar cambios significativos, ya que si ellos no se sienten identificados con la empresa podría provocar un desequilibrio y no ayudar en la solución 0 en las mejoras a implementar.

Si la empresa decide no prepararse con anterioridad, o sea, prefiere ser reactivo antes que proactivo, decidirá en el momento justo tomar medidas respecto a la situación que los aqueja. Una de las alternativas más drásticas es disminuir los sueldos de todos los trabajadores de la empresa y, a su vez, es un desafío motivar a todos para que apoyen esta decisión. Es un desafío, ya que los conductores fueron muy claros al momento de contestar esta pregunta. La gran mayoría comentó que nunca aceptarían esta medida, pero la historia de la empresa dice lo contrario. Uno de los entrevistados comentó que hace algunos años la empresa tomó esta decisión y todos la aceptaron. Se efectuaron charlas informativas explicando los antecedentes de la situación y los beneficios que traería para todos el acogerse a ella. Estas actividades informativas permitieron que esta medida se realizara. Fue un trabajo en equipo, todos colaboraron para enfrentar y salir triunfantes de la situación. Si decide realizar otra de las actividades clasificadas en la gestión como la reducción 
de personal la empresa, debe ser prudente ya que son distintas formas de actuar, pero siempre manteniendo la característica de ser reactiva y lo que no es bueno.

Se recomienda que la empresa sea precavida y utilice el mejoramiento continuo de procesos, en un mismo porcentaje los recursos utilizados, el tiempo y la calidad. No es necesario que se ponga de relieve todos los meses, pero si una vez al año. Replantearse si lo que se está haciendo está bien 0 es mejor buscar nuevas maneras de trabajar, para así ir avanzando de manera creciente, preocupándose realmente de sus trabajadores, ya que la empresa estará preparada desde antes y no estará entre sus alternativas desvincular una cifra considerable de trabajadores, en momentos de angustia y desesperación. Esta no es una alternativa para la empresa, por lo que se asume que acogerán las propuestas entregadas en esta investigación. 


\section{BIBLIOGRAFÍA}

- Chase, Jacobs y Aquilano (2005) Administración de la producción y operaciones para una ventaja competitiva. México: Mc Graw-Hill.

- Chiavenato, I (2002) Gestión del talento humano. Bogotá, Colombia: Mc Graw-Hill.

- Dale, H (2009) Control de calidad. México: Pearson, Prentice Hall.

- $\quad$ EFE. (2011, Noviembre 14). Expertos creen que Latinoamérica resistirá la crisis pero debe ser cautelosa. Diario financiero online.

- Gaither y Frazier (2000). Administración de producción y operaciones. México: International Thomson Editores

- Gaither y Frazier (2000). Administración de producción y operaciones. México: International Thomson Editores

- $\quad$ Gomez, Balkin y Cardy (2001) Dirección y gestión de recursos humanos. España: Pearson, Prentice-Hall.

- Hensen y Mowen (2003) Administración de costos, contabilidad y control. México: Thomson, Learning.

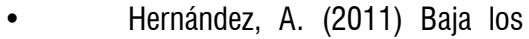
costos de operación de tu empresa. Alto Nivel.

- $\quad$ Hitt, Black y Porter (2006)

Administración. México: Pearson Prentice Hall.

- Horngren, Sundem, y Selto (1994) Contabilidad administrativa. México: Pearson, Prentice Hall.

- Jones, Werner, Terrel y Terrel (2001) Introducción a la contabilidad administrativa, perspectiva del usuario. Colombia: Prentice Hall.

Krajewski y Ritzman (2000).
Administración de operaciones, estrategia y análisis. México: Prentice hall.

- Livacic, C. (2009) La Comunicación y la Información como sistema de relaciones y logro al interior de las Organizaciones. Revista de Estudios Politécnicos, Vol. VII, $\mathrm{n}^{0} 11$. Recuperado de http://www.scielo. oces.mctes.pt/pdf/tek/n11/n11a12.pdf

- Mariña, M. (2007), Comportamiento Organizacional. Recuperado de

http://ocw.uni.edu.pe/ocw/facultad-deingenieria-industrial-y-sistemas/desarrolloorganizacional/lecturas/Lec15.pdf

- $\quad$ Pin, J. Los despidos masivos se deben a la falta de previsión e imaginación. (Entrevista con expertos) Recuperado de http://www.laboris.net/ static/em_opinion_jose-ramon-pin.aspx

- Samuel, C (2001) Administración Moderna. Bogotá. Colombia: Pearson, Prentice hall.

- $\quad$ Schroeder (2005) Administración de operaciones, concepto y casos contemporáneos. México: Mc Graw Hill.

- Urdaneta, M. (2008) La responsabilidad social en la industria farmacéutica del Estado de Zulia. Revista Venezolana de Gerencia. vol.13, no.41 p.49-72. Recuperado dehttp://www. sciel0.org.ve/scielo.php?pid =S1315$\underline{99842008000100004 \& \text { script }=\text { sci arttext }}$

- Zúñiga, R. Operaciones: concepto, sistema, estrategia y simulación. (Revista Latinoamericana de Administración) Recuperado de http://redalyc.uaemex.mx/ pdf/716/71603402.pdf 